\title{
Silicon crystalline undulator prototypes: Manufacturing and x-ray characterization
}

\author{
Riccardo Camattari, ${ }^{1, *}$ Laura Bandiera, ${ }^{1}$ Viktor Tikhomirov, ${ }^{2}$ Marco Romagnoni, ${ }^{3,1}$ \\ Enrico Bagli, ${ }^{1}$ Giacomo Germogli, ${ }^{1}$ Alexei Sytov, ${ }^{1}$ Tais Maiolino, ${ }^{1}$ Melissa Tamisari, ${ }^{4}$ \\ Andrea Mazzolari, ${ }^{1}$ Vincenzo Guidi, ${ }^{3}$ and Gianluca Cavoto, ${ }^{5,6}$ \\ ${ }^{1}$ INFN, Section of Ferrara, Via Saragat 1, 44122 Ferrara, Italy \\ ${ }^{2}$ Institute for Nuclear Problems, Belarusian State University, Minsk 220030, Belarus \\ ${ }^{3}$ Department of Physics and Earth Sciences, University of Ferrara, Via Saragat 1/c, 44122 Ferrara, Italy \\ ${ }^{4}$ Dipartimento di Scienze biomediche e chirurgico specialistiche, \\ Via Fossato di Mortara, 64/A-44121 Ferrara, Italy \\ ${ }^{5}$ INFN, Section of Rome, Piazzale Aldo Moro 2, 00185 Rome, Italy \\ ${ }^{6}$ Sapienza, Università degli Studi di Roma, Piazzale Aldo Moro 2, 00185 Rome, Italy
}

(Received 11 January 2019; published 24 April 2019)

\begin{abstract}
Short-period crystalline undulators have the potential to enable intense and monochromatic sources of hard $\mathrm{X}$ and $\gamma$ radiation. Three crystalline undulators were manufactured and their structural properties were characterized at the ESRF x-ray light source. In particular, a crystalline undulator consists in a periodically bent crystal in which channeled electrons or positrons follow the bending of the crystalline planes, thus generating electromagnetic radiation in analogy with standard magnetic undulators. State-of-the-art magnetic undulators can be built with a period of no less than a few $\mathrm{cm}$. On the other hand, a crystalline undulator could be built with a period down to the sub-mm range. Thus, a crystalline undulator would allow generating harder photons. Since the radiator consists of a small crystal, a crystalline undulator is a valid and low-cost alternative for intense hard radiation production at electron accelerator facilities. The three samples were realized at the INFN laboratory of Ferrara, Italy, through the grooving method, namely a method developed to induce a self-standing and adjustable deformation to a crystal. The samples showed a uniform and sinusoidal curvature, thus proving that the grooving method is suitable for building crystalline undulators.
\end{abstract}

DOI: 10.1103/PhysRevAccelBeams.22.044701

\section{INTRODUCTION}

As a charged particle impinges on the atomic planes of a crystal at a sufficiently small angle with respect to the planes, it can be channeled inside the crystalline interplanar potential [1]. In 1976, Tsyganov proposed to use channeling in bent crystals for particle beams steering [2]. This effect has been investigated for years and recently exploited to steer the highest-energy beam worldwide, i.e., the 6.5 $\mathrm{TeV}$ proton beam at the LHC [3].

Channeling in a periodically bent crystal with a sinusoidal-like deformation, i.e., a crystalline undulator (CU), has been theoretically investigated as a tool for production

\footnotetext{
"Present address: INFN, Section of Ferrara, Via Saragat 1, 44122 Ferrara, Italy.

camattari@fe.infn.it.

Published by the American Physical Society under the terms of the Creative Commons Attribution 4.0 International license. Further distribution of this work must maintain attribution to the author(s) and the published article's title, journal citation, and DOI.
}

of intense and monochromatic electromagnetic radiation $[4,5]$. The idea of a CU was conceived in the late 1970s by Baryshevsky et al. [6] and developed by [7,8]. In fact, a $\mathrm{CU}$ mimes the magnetic undulators owing to the strong crystalline interplanar electrostatic field $\left(10^{9} \mathrm{~V} / \mathrm{cm}\right)$, which replaces the role of magnets to force particle oscillation (see Fig. 1). In such a system, in addition to a broadband channeling radiation background [4], there appears an intense and monochromatic undulator-like radiation due to the periodic motion of particles that follow the planes bending.

In a magnetic undulator, the period of oscillations $\lambda_{u}$ is limited to no less than $1 \mathrm{~cm}$, while in a $\mathrm{CU} \lambda_{u}$ can go down to the sub-mm range. The wavelength $(\lambda)$ of the $\gamma$ rays emitted on axis is given by

$$
\lambda=\frac{\lambda_{u}}{2 \gamma^{2}}\left(1+k^{2} / 2\right)
$$

where $\gamma$ the Lorentz factor of the beam impinging the CU, and $k$ the so-called undulator strength parameter 


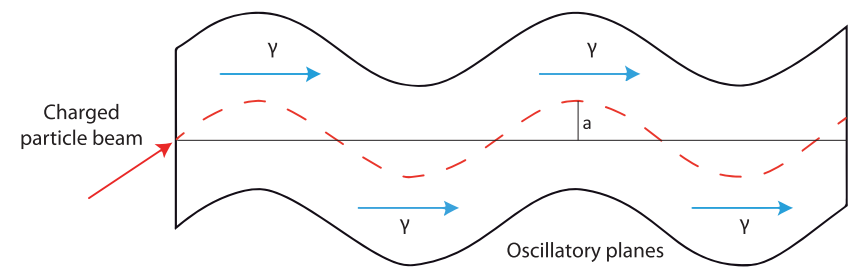

FIG. 1. Schematic representation of a crystalline undulator (CU). The red dashed line represents the trajectory of the channeled beam. The amplitude $A$ of the deformation of the oscillatory planes is highlighted.

$$
k=\frac{e B \lambda_{u}}{2 \pi m_{e} c},
$$

where $\mathrm{B}$ is the magnetic field, $m_{e}$ the electron mass, and $c$ the speed of light.

Thus, a CU would allow generating harder photons with respect to a magnetic undulator. In particular, a CU with period of the order of $10^{1}-10^{2} \mu \mathrm{m}$ can generate $\mathrm{MeV}$ photons using a $\mathrm{GeV}$ electron beam.

A source of $\mathrm{MeV}$ radiation can be realized by other methods. The most promising method is the inverse Compton scattering (ICS). In an ICS source, an intense laser beam of visible or near-infrared photons is scattered off an electron beam with typically a few hundred $\mathrm{MeV}$ energy. However, ICS requires the usage of high-intensity lasers, while a CU-based source would eliminate the need for a laser, thereby strongly diminishing its cost. An ICS source can use a very short laser wavelength, below $1 \mu \mathrm{m}$. In principle, also a $\mathrm{CU}$ can reach submicrometric period, since there exist enabling technologies, as described in the work of Kostyuk [5]. The most mature of them is the growing of $\mathrm{Si}_{1-x} \mathrm{Ge}_{x}$ crystals [9] with a periodically varying Ge content $x$ [10].

Over the years, different studies aimed at demonstrating the feasibility of a CU were worked out [7,11]. A first manufacturing technique was based on patterned ion implantation by swift ions. In particular, it was demonstrated that implantation of $150 \mathrm{keV} \mathrm{He} \mathrm{He}^{+}$ions can be exploited for $\mathrm{CU}$ fabrication [12]. Another technique consisted of patterning a thin film of strained silicon nitride on a silicon monocrystal to generate an undulated structure [13]. Another technique consisted on sandblasting the two major surfaces of a crystal to produce a patterned amorphized layer capable of undulating the sample [14]. Sandblasting is a mechanical process consisting in driving a stream of abrasive material against a sample through a pressurized fluid, usually compressed air. This technique permits to bend from thin to thick crystals, up to a few $\mathrm{mm}$. As said previously, the most mature method is based on epitaxial growth of Si-Ge crystalline alloy layers on silicon substrates [15-17]. Due to the different lattice constants of $\mathrm{Si}$ and $\mathrm{Ge}$, growing a $\mathrm{Si}_{1-x} \mathrm{Ge}_{x}$ on a $\mathrm{Si}$ substrate by periodically varying the " $x$ " value during the growth results in an undulated shape of the crystalline planes. This latter method was exploited at the MAinzer MIkrotron (MAMI) for the currently most convincing evidence of $\mathrm{CU}$ radiation, where hard radiation generated by sub-GeV electrons interacting with a micrometric $\mathrm{CU}$ was observed.

Another procedure for the realization of a $\mathrm{CU}$ is the grooving method [18]. This method consists of making a series of grooves on the major surfaces of a crystal. Indeed, it was shown that a series of grooves may cause a permanent and reproducible deformation of the whole sample $[19,20]$. The plasticization that occurs in the thin superficial layer transfers an elastic strain field to the crystal bulk, producing a deformation field within the crystal itself. The realization of a millimetric or even a submillimetric undulator could be achieved by making an alternate pattern of parallel grooves on both the surfaces of a crystal. This method has the main advantage that no contaminating materials are added to the sample, thus the obtained $\mathrm{CU}$ is made only of the starting pure material. Moreover, since the surfaces result to be plasticized, the deformation turns out to be self-standing. Such a method was used to realize optical elements for hard x-ray focusing [21,22] and for the manufacturing of the first prototype of grooved $\mathrm{CU}$, which was tested with a $400-\mathrm{GeV} / c$ proton beam at CERN [23]. In this case, a homogeneous deformation was obtained, with $\lambda_{u}=1.0 \mathrm{~mm}$.

In this paper, the realization and the structural characterization of three $\mathrm{CU}$ samples, obtained via the grooving method, featuring a period $\lambda_{u}$ ranging from 334 down to $80 \mu \mathrm{m}$, are shown, which is an interesting range for applications. The CU samples were realized at the INFN laboratory of Ferrara, Italy, while their structural characterization was performed via $\mathrm{x}$-ray diffraction at the ID11 line at the European Synchrotron Radiation Facility (ESRF) in Grenoble (France), using a hard x-ray beam tuned to $100 \mathrm{keV}$. The samples showed a uniform and sinusoidal curvature.

\section{EXPERIMENTAL METHOD}

Three Si samples were shaped as rectangular frames with size $10.0 \times 15.0 \times 0.2 \mathrm{~mm}^{3}$, featuring a little protrusion in one corner, as can be seen in Fig. 2. The frame was diced starting from high pure $\mathrm{Si}$ (111) wafer 0.2-mm thick by means of a diamond blade through the usage of a DISCO DAD 3220 dicing machine. Then, an alternate pattern of parallel grooves was produced on both surfaces of the protrusion. The depth of the grooves was $50 \mu \mathrm{m}$ for each side. Indeed, the actual CU is only the protrusion visible on the top-right corner of Fig. 2.

The width of the grooves, the distance between consecutive grooves, and the width of the undulator were varied for the three samples. In particular, the values of the distance between consecutive grooves correspond to the $\lambda_{u}$ of the CUs and were set to be twice the width of the blade 


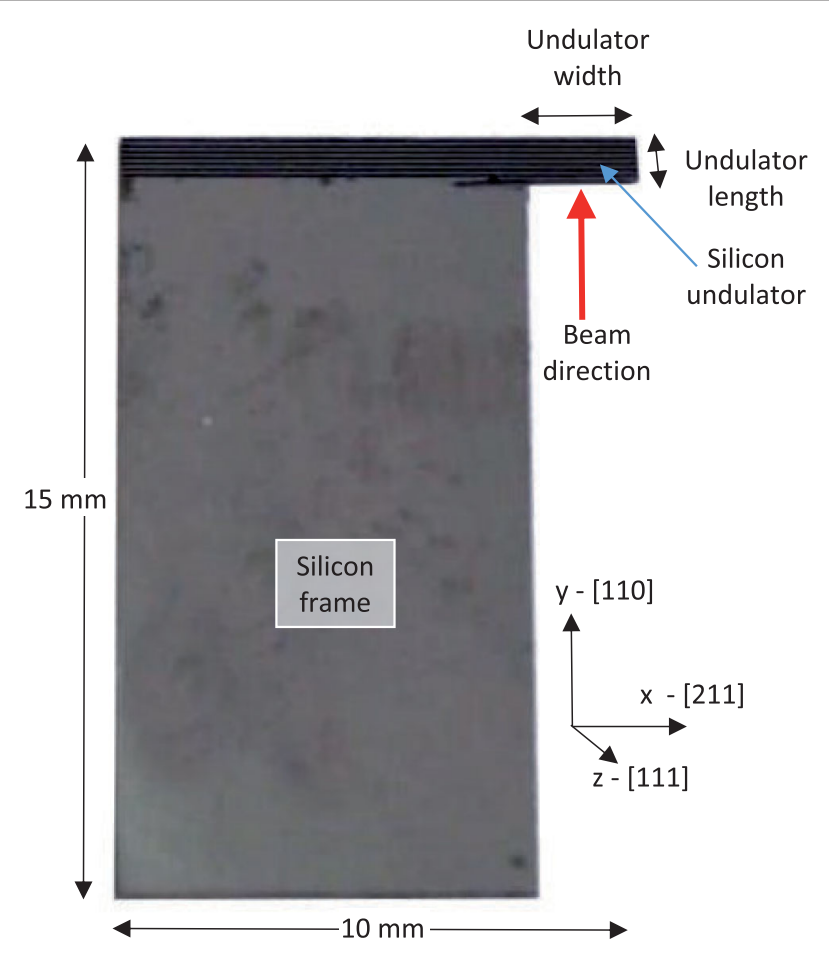

FIG. 2. Pictures of a CU obtained through the grooving method. The direction of the charged particle beam that would be channeled into the $\mathrm{CU}$ is highlighted with a red arrow.

used for the grooves. All the values of the manufacturing process are listed in Table I.

The three CUs were successively tested through x-ray diffraction at the ID11 beam line of ESRF. A highly monochromatic and quasiparallel beam was tuned to $100 \mathrm{keV}$ energy. The monochromaticity was of the order of $\Delta E / E=10^{-3}$. The beam was $100 \times 5 \mu \mathrm{m}^{2}$. The beam was directed along the $z$ axis, entered the crystal protrusion from one of its surfaces, and diffracted on the (220) planes, namely the planes perpendicular to the $y$ axis, thus traversing the crystal (i.e. in Laue geometry). (220) planes

TABLE I. Features of the crystalline undulators.

\begin{tabular}{lccc}
\hline \hline & Sample 1 & Sample 2 & Sample 3 \\
\hline Material & Silicon & Silicon & Silicon \\
Channeling plane & $(111)$ & $(111)$ & $(111)$ \\
Channeling axis & $\langle 110\rangle$ & $\langle 110\rangle$ & $\langle 110\rangle$ \\
Frame width- $x[\mathrm{~mm}]$ & 10 & 10 & 10 \\
Frame length $-y[\mathrm{~mm}]$ & 15 & 15 & 15 \\
Frame thickness-z $[\mathrm{mm}]$ & 0.2 & 0.2 & 0.2 \\
CU width- $x[\mathrm{~mm}]$ & 2 & 2 & 2 \\
CU length-y $[\mathrm{mm}]$ & 3.34 & 0.88 & 0.80 \\
CU thickness- $z[\mathrm{~mm}]$ & 0.2 & 0.2 & 0.2 \\
Number of periods & 10 & 7 & 11 \\
Periods $\left(\lambda_{u}\right)(\mu \mathrm{m})$ & 334 & 120 & 80.0 \\
Blade width $(\mu \mathrm{m})$ & 160 & 60 & 40 \\
Groove depth $(\mu \mathrm{m})$ & 50 & 50 & 50 \\
\hline \hline
\end{tabular}

TABLE II. Experimental parameters.

\begin{tabular}{lc}
\hline \hline Beam energy $(\mathrm{keV})$ & 100 \\
Beam size $\left(\Delta_{x} \times \Delta_{y}, \mu \mathrm{m}^{2}\right)$ & $100 \times 5$ \\
Beam monochromaticity $(\Delta E / E)$ & $10^{-3}$ \\
\hline \hline
\end{tabular}

were used because (110) planes correspond to a forbidden diffraction.

The characterization of the samples was carried out by performing rocking curves (RCs), i.e., the transmitted and the diffracted beam intensities were recorded while the crystal was being rotated around the position at which Bragg condition was met, i.e. the sample was rotated around the $x$ axis. The sample holder was set far enough from the detectors to allow sufficient separation of the diffracted and transmitted beams.

Bragg diffraction occurs if the angle $(\theta)$ between the $\mathrm{x}$-ray beam and the diffracting planes follows the Bragg's law, $2 d_{h k l} \sin (\theta)=\lambda$, where $d_{h k l}$ is the d-spacing of planes (hkl) and $\lambda$ the wavelength of the x-ray beam. Thus, a RC gives as output the angular orientation of the diffracting planes. Since the width of the $\mathrm{x}$-ray beam along the $y$ axis was $\Delta_{y}=5 \mu \mathrm{m}$, the measurement permitted to record the angle of the planes perpendicular to the $y$ axis with a good spatial resolution $\left(\Delta_{y} \ll \lambda_{u}\right)$. Indeed, the x-ray beam was collimated to the minimum size along the $y$ axis to increase the precision of the measurement. On the contrary, the size of the beam along the $x$ axis was set to be $100 \mu \mathrm{m}$ to increase the flux of the x-ray beam. The experimental parameters are listed in Table II.

A large series of RCs were recorded as a function of the position along the $\mathrm{CU}$ length ( $y$ axis), to characterize the periodical deformation of the whole $\mathrm{CU}$ length.

In order to subtract the systematical errors due to the instrumental tolerances of the movements of the goniometer on which the CUs were placed for the measurements, a flat reference crystal with identical crystallographic orientation was fixed behind the CUs. Thus, the (220) diffraction peak of the reference crystal was recorded together with the peak of the CU. Both the angular position of the $\mathrm{CU}$ diffraction peaks and the reference peaks were recorded as a function of the impact position of the x-ray beam on the undulator. Since the peak positions of the flat reference sample should not move as a function of $y$, the stage errors can be compensated by taking into account the reference peak positions.

\section{EXPERIMENTAL RESULTS}

The experimental results of the recorded RCs are shown in Fig. 3 for sample 1 (a), sample 2 (b), and sample 3 (c). Beam divergence, acquisition time, and beam size were taken into account in the data analysis, as well as the systematical errors due to the goniometer motors. For every sample of Fig. 3, the upper part of the plot corresponds to 

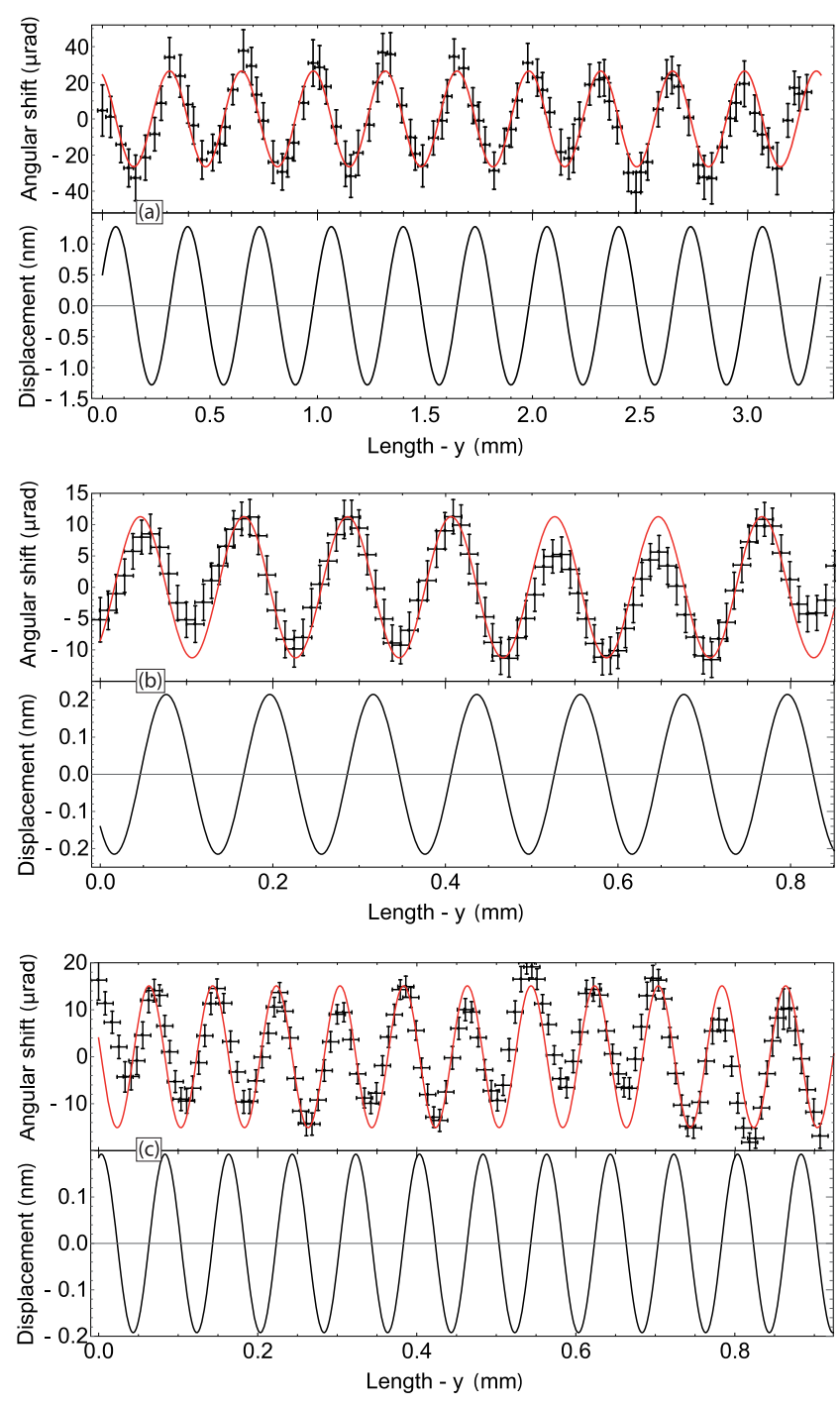

FIG. 3. Angular shift and deformation of the crystallographic planes due to the grooving process along the undulator length, measured through hard monochromatic x-ray diffraction. The RCs were fitted using a sinusoidal function (plotted in red). The deformation of the crystallographic planes was obtained through numerical integration of the fitted RCs. (a), (b), and (c) correspond to sample 1 , sample 2 , and sample 3 , respectively.

the direct measurements of the diffracting angles (RCs) as a function of the position along the undulator length. In particular, in these plots, every point represents the peak of the RCs, taking into account the position of the reference peak to eliminate the systematic errors of the movement stage. Then, the RCs were fitted using a sinusoidal function, which is plotted in red in the figure. Such functions were integrated to obtain the deformation of the crystallographic planes as a function of the position along the undulator length. The function used for the fit was $A \sin \left(\frac{2 \pi\left(y-\phi_{y}\right.}{\lambda_{u}}\right)$, where $A$ is the undulation amplitude and $\phi_{y}$ the phase. The integrated functions are shown below the respective RC plots in Fig. 3.
TABLE III. Features of the crystalline undulators.

\begin{tabular}{lccc}
\hline \hline & Sample 1 & Sample 2 & Sample 3 \\
\hline Periods $\left(\lambda_{u}\right)(\mu \mathrm{m})$ & 334 & 120 & 80.0 \\
Amplitude $(A)[\mathrm{nm}]$ & 1.28 & 0.20 & 0.15 \\
Strength parameter $(\mathrm{k})$ & 0.46 & 0.20 & 0.23 \\
\hline \hline
\end{tabular}

It was then possible to obtain the amplitude $(A)$ and the strength parameter $(k)$ of the realized undulator. The values are listed in Table III and range from 1.28 to $0.15 \mathrm{~nm}$ for the amplitude and from 0.46 to 0.20 for the strength parameter. It is important to notice that the amplitude of the deformation decreases proportionally with the width of the grooves, because the stress due to the grooves per unit of surface is constant. Therefore, if the width of the groove decreases, also the amplitude of the deformation decreases. Thus, the more $\lambda_{u}$ is reduced, the more the undulation amplitude is decreased.

\section{DISCUSSION AND CONCLUSIONS}

Three crystalline undulators were produced and characterized via hard $\mathrm{x}$-ray diffraction. The deformation turned out to be homogeneous throughout the samples, highlighting that the grooving method is capable of inducing a controlled and adjustable deformation to the crystals. Since the deformation is in the elastic regime [24], the whole thickness of the undulators is deformed as shown in Fig. 3. Indeed, the x-ray measurements investigated the whole thickness of the samples, since the beam was in Laue geometry. However, since the groove depth was $50 \mu \mathrm{m}$ and the sample thickness was $200 \mu \mathrm{m}$, the active part of the samples for channeling is $100 \mu \mathrm{m}$ thick. A real positron beam could impinge the whole undulator thickness. However, if the beam hit the grooved part of the sample, some additional background is expected.

Hard $\mathrm{x}$-ray diffraction indicates that the crystalline quality of the samples was preserved. However, channeling with charged particles is mandatory to attest that the crystallographic quality was preserved enough to permit highefficiency channeling and thus intense $\gamma$ ray production.

This study is aimed to examine the possibility to obtain a self-standing $\mathrm{CU}$ made of pure material through the grooving method. Such a procedure has the advantages of producing a $\mathrm{CU}$ with no need of an external holder for the deformation, and the crystal has no contaminant materials inside. On the other hand, the achievable deformation is limited by the maximum stress that can be imparted through the grooves, namely about $40 \mathrm{MPa}$ [25]. Therefore, the maximum amplitude decreases if the undulator period is decreased.

The manufactured prototypes are ready to be tested with positron beams. In particular, sample 1 can be tested with positron beam with energy $E \simeq 10 \mathrm{GeV}$. Indeed, for such beam, the dechanneling length $L_{D}$ is about $5 \mathrm{~mm}[7,26]$. 
Thus, the whole $\mathrm{CU}$ can be crossed by the positrons in channeling condition, since the number of periods that can be traveled is $L_{D} / \lambda_{u} \simeq 15$. Furthermore, the amplitude of the $\mathrm{CU}$ is larger than the interplanar spacing $(A>d)$, as required to achieve a feasible $\mathrm{CU}$ in the large amplitude large period regime [11,27-30]. The authors of [11,27-30] introduced the $C$ parameter, defined as

$$
C=\frac{4 \pi^{2} E a}{U_{\max }^{\prime} \lambda_{u}^{2}}
$$

where $U_{\max }^{\prime}$ is the maximal planar field and holds $5.37 \mathrm{GeV} / \mathrm{cm}$ for $\mathrm{Si}$ (111) in the Doyle-Turner approximation. It should be $0.01<C<0.2$ to have an intense photon production via the undulator. For sample 1, $C \simeq 0.01$, while samples 2 and $3 C$ turns out to be too small, $\simeq 0.001$.

The photons generated by sample 1 should have an energy that can be roughly estimated through Eqs. (1) and (2), and turns out to be $\sim 2-3 \mathrm{MeV}$. A better estimation of the emitted radiation by this CU sample was worked out through a Monte Carlo code [8,31,32] based on the Baier Katkov quasiclassical method [33]. In the simulation, the Ter-Mikaelian density effect and the transition radiation were also taken into account. The distribution of the radiation emission probability generated by a $10-\mathrm{GeV}$ positron beam interacting with the sample is shown in Fig. 4. The beam divergence was set to $30 \mu \mathrm{rad}$. The radiation emission probability was averaged over a large number of particle trajectories and photon emission angles. The emission spectrum has a sharp peak at $\sim 1.5 \mathrm{MeV}$. Since the channeling radiation (CR) is expected in the $50 \mathrm{MeV}$ region [33], the $\mathrm{CR}$ and the $\mathrm{CU}$ peaks have no overlap. In the energy range from 0.5 to $2 \mathrm{MeV}$, the peak of the $\mathrm{CU}$ radiation results to be more than 20 times higher than for a $\mathrm{Si}$ amorphous sample of the same thickness.

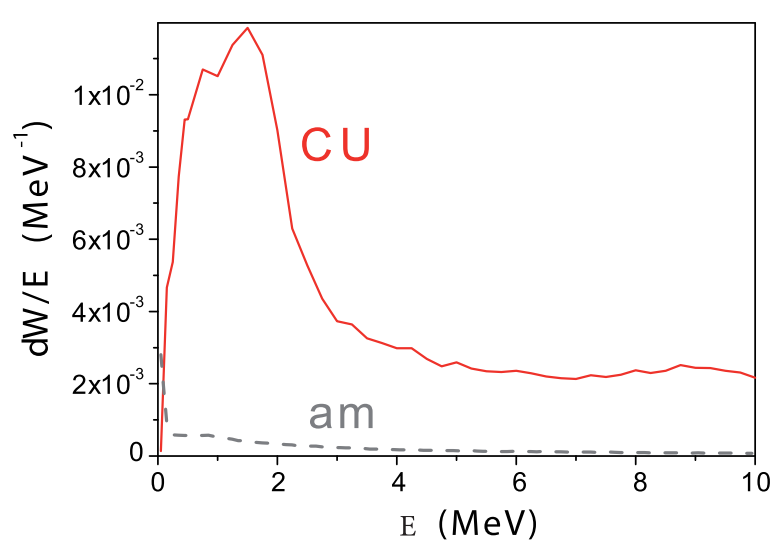

FIG. 4. Simulated radiation emission probability for a $10-\mathrm{GeV}$ positron beam interacting with sample 1 (solid line) and the Bethe-Heitler amorphous emission probability for a Si sample of the same thickness (dashed line). The radiation collimation half-angle is $0.5 / \gamma, \gamma$ being the Lorentz factor.
Samples 2 and 3 have a shorter period, but their amplitude is limited to be less than $0.2 \mathrm{~nm}$, thus $A<d$. Given the very small amplitude and the small value of $C$, samples 2 and 3 cannot show a significant undulation radiation. However, these samples are shown in this paper to describe the possibility and the limitation of the grooving technique as a tool for the manufacturing of optimized CU. In order to increase the amplitude keeping the period fixed, an experimental procedure to increase the stress produced by the grooves has to be studied.

Once verified, the hard, intense, and monochromatic radiation of a $\mathrm{CU}$ could enable important experiments in a wealth of research fields, such as Physics, Medicine, Biology, and Material Science.

\section{ACKNOWLEDGMENTS}

We acknowledge the financial support from 7th Framework Programme-European Research Council Ideas Consolidator Grant No. 615089 CRYSBEAM and the partial support by the European Commission through the PEARL Project within the Horizon 2020-Marie Skłodowska-Curie actions-Research And Innovation Staff Exchange-2015 call, Grant Agreement No. 690991. We also acknowledge partial support of the Istituto Nazionale di Fisica Nucleare under Grant No. 73-Oriented Scintillator CRystals and the Commissione Scientifica Nazionale 5-ELIOT projects. We also acknowledge the CINECA award under the ISCRA initiative, for the availability of high performance computing resources and support. A. Mazzolari and V. Guidi acknowledge founding from Progetti di Rilevante Interesse Nazionale 2015LYYXA8 "Multi-scale mechanical models for the design and optimization of micro-structured smart materials and metamaterials."

[1] J. Lindhard, Influence of crystal lattice on motion of energetic charged particles, Mat. Fys. Medd. K. Dan. Vidensk. Selsk 34, 1 (1965).

[2] E. N. Tsyganov, Some aspects of the mechanism of a charge particle penetration through a monocrystal, Fermilab Technical Report No. TM-682, 1976.

[3] W. Scandale et al., Observation of channeling for $6500 \mathrm{GeV} / c$ protons in the crystal assisted collimation setup for LHC, Phys. Lett. B 758, 129 (2016).

[4] V. Baryshevskii and V. Tikhomirov, Synchrotron-type radiation processes in crystals and polarization phenomena accompanying them, Sov. Phys. Usp. 32, 1013 (1989).

[5] A. Kostyuk, Crystalline Undulator with a Small Amplitude and a Short Period, Phys. Rev. Lett. 110, 115503 (2013).

[6] V. Baryshevsky, A. Grubich, and I. Y. Dubovskaya, Generation of $\gamma$-quanta by channeled particles in the presence of a variable external field, Phys. Lett. A 77, 61 (1980).

[7] A. V. Korol, A. V. Solov'yov, and W. Greiner, Channeling and Radiation in Periodically Bent Crystals (Springer, New York, 2013), Vol. 69. 
[8] V. Baryshevsky and V. Tikhomirov, Crystal undulators: from the prediction to the mature simulations, Nucl. Instrum. Methods Phys. Res., Sect. B 309, 30 (2013).

[9] M. Breese, Beam bending using graded composition strained layers, Nucl. Instrum. Methods Phys. Res., Sect. B 132, 540 (1997).

[10] U. Mikkelsen and E. Uggerhøj, A crystalline undulator based on graded composition strained layers in a superlattice, Nucl. Instrum. Methods Phys. Res., Sect. B 160, 435 (2000).

[11] A. V. Korol, A. V. Solov'Yov, and W. Greiner, Total energy losses due to the radiation in an acoustically based undulator: the undulator and the channeling radiation included, Int. J. Mod. Phys. E 09, 77 (2000).

[12] V. Bellucci, R. Camattari, A. Mazzolari, G. Paternò, V. Guidi, C. Scian, G. Mattei, and L. Lanzoni, Ion implantation for manufacturing bent and periodically bent crystals, Appl. Phys. Lett. 107, 064102 (2015).

[13] V. Guidi, L. Lanzoni, A. Mazzolari, G. Martinelli, and A. Tralli, Design of a crystalline undulator based on patterning by tensile $\mathrm{Si}_{3} N_{4}$ strips on a Si crystal, Appl. Phys. Lett. $\mathbf{9 0}$, 114107 (2007).

[14] R. Camattari, G. Paternò, M. Romagnoni, V. Bellucci, A. Mazzolari, and V. Guidi, Homogeneous self-standing curved monocrystals, obtained using sandblasting, to be used as manipulators of hard X-rays and charged particle beams, J. Appl. Crystallogr. 50, 145 (2017).

[15] T. N. Wistisen, K. K. Andersen, S. Yilmaz, R. Mikkelsen, J. L. Hansen, U. I. Uggerhøj, W. Lauth, and H. Backe, Experimental Realization of a New Type of Crystalline Undulator, Phys. Rev. Lett. 112, 254801 (2014).

[16] H. Backe, D. Krambrich, W. Lauth, K. Andersen, J. Hansen, and U. Uggerhøj, J. Phys. Conf. Ser. 438, 012017 (2013).

[17] H. Backe, D. Krambrich, W. Lauth, K. Andersen, J. Hansen, and U. Uggerhøj, Radiation emission at channeling of electrons in a strained layer $S i_{1-x} G e_{x}$ undulator crystal, Nucl. Instrum. Methods Phys. Res., Sect. B 309, 37 (2013).

[18] S. Bellucci, S. Bini, V. M. Biryukov, Y. A. Chesnokov, S. Dabagov, G. Giannini, V. Guidi, Y. M. Ivanov, V. I. Kotov, V. A. Maisheev, C. Malagù, G. Martinelli, A. A. Petrunin, V. V. Skorobogatov, M. Stefancich, and D. Vincenzi, Experimental Study for the Feasibility of a Crystalline Undulator, Phys. Rev. Lett. 90, 034801 (2003).

[19] N. Barrière, V. Guidi, V. Bellucci, R. Camattari, T. Buslaps, J. Rousselle, G. Roudil, F.-X. Arnaud, P. Bastie, and L. Natalucci, High diffraction efficiency at hard X-ray energy in a silicon crystal bent by indentation, J. Appl. Crystallogr. 43, 1519 (2010).
[20] V. Bellucci, R. Camattari, V. Guidi, I. Neri, and N. Barrière, Self-standing bent silicon crystals for very high efficiency Laue lens, Exp. Astron. 31, 45 (2011).

[21] R. Camattari, A. Battelli, V. Bellucci, and V. Guidi, Highly reproducible quasi-mosaic crystals as optical components for a Laue lens, Exp. Astron. 37, 1 (2014).

[22] V. Liccardo, E. Virgilli, F. Frontera, V. Valsan, E. Buffagni, C. Ferrari, E. Bonnini, A. Zappettini, V. Guidi, V. Bellucci, and R. Camattari, Study and characterization of bent crystals for Laue lenses, Exp. Astron. 38, 401 (2014).

[23] E. Bagli, L. Bandiera, V. Bellucci, A. Berra, R. Camattari, D. De Salvador, G. Germogli, V. Guidi, L. Lanzoni, D. Lietti, A. Mazzolari, M. Prest, V. Tikhomirov, and E. Vallazza, Experimental evidence of planar channeling in a periodically bent crystal, Eur. Phys. J. C 74, 3114 (2014).

[24] Y. V. Milman, I. V. Gridneva, and A. A. Golubenko, Construction of Stress-Strain Curves for Brittle Materials by Indentation in a Wide Temperature Range, Sci. Sintering 39, 67 (2007).

[25] R. Camattari, V. Guidi, L. Lanzoni, and I. Neri, Experimental analysis and modeling of self-standing curved crystals for focusing of X-rays, Meccanica 48, 1875 (2013).

[26] V. Biryukov, Y. A. Chesnokov, and V. I. Kotov, Crystal Channeling and Its Application at High-Energy Accelerators (Springer, Berlin, 1997).

[27] A. V. Korol, A. V. Solov'yov, and W. Greiner, Coherent radiation of an ultrarelativistic charged particle channelled in a periodically bent crystal, J. Phys. G 24, L45 (1998).

[28] A. V. Korol, A. V. Solov'yov, and W. Greiner, Channeling of positrons through periodically bent crystals: On the feasibility of crystalline undulator and gamma-laser, Int. J. Mod. Phys. E 13, 867 (2004).

[29] A. V. Korol, A. V. Solov'yov, and W. Greiner, The influence of the dechannelling process on the photon emission by an ultra-relativistic positron channelling in a periodically bent crystal, J. Phys. G 27, 95 (2001).

[30] W. Krause, A. V. Korol, A. V. Solov'yov, and W. Greiner, Total spectrum of photon emission by an ultra-relativistic positron channelling in a periodically bent crystal, J. Phys. G 26, L87 (2000).

[31] V. Guidi, L. Bandiera, and V. Tikhomirov, Radiation generated by single and multiple volume reflection of ultrarelativistic electrons and positrons in bent crystals, Phys. Rev. A 86, 042903 (2012).

[32] L. Bandiera et al., On the radiation accompanying volume reflection, Nucl. Instrum. Methods Phys. Res., Sect. B 309, 135 (2013).

[33] V. Baier, V. Katkov, and V. Strakhovenko, Electromagnetic Processes at High Energies in Oriented Single Crystals (World Scientific, Singapore, 1998). 